\title{
ARTICLE OPEN Doping induced Mott collapse and possible density wave instabilities in $\left(\mathrm{Sr}_{1-\mathrm{x}} \mathrm{La}_{\mathrm{x}}\right)_{3} \mathrm{Ir}_{2} \mathrm{O}_{7}$
}

\author{
Zhenyu Wang ${ }^{1}$, Daniel Walkup ${ }^{2,3}$, Yulia Maximenko ${ }^{1}$, Wenwen Zhou $^{2}$, Tom Hogan ${ }^{2,4}$, Ziqiang Wang ${ }^{2}$, Stephen D. Wilson ${ }^{4}$ and \\ Vidya Madhavan ${ }^{1}$
}

The path from a Mott insulating phase to high temperature superconductivity encounters a rich set of unconventional phenomena involving the insulator-to-metal transition (IMT), such as emergent electronic orders and pseudogaps, that ultimately affect the condensation of Cooper pairs. A huge hindrance to understanding the origin of these phenomena is the difficulty in accessing doping levels near the parent state. The $J_{\text {eff }}=1 / 2$ Mott state of the perovskite strontium iridates has revealed intriguing parallels to the cuprates, with the advantage that it provides unique access to the Mott transition. Here, we exploit this accessibility to study the IMT and the possible nearby electronic orders in the electron-doped bilayer iridate $\left(\mathrm{Sr}_{1-x} \mathrm{La}_{\mathrm{x}}\right)_{3} \mathrm{Ir}_{2} \mathrm{O}_{7}$. Using spectroscopic imaging scanning tunneling microscopy, we image the La dopants in the top as well as the interlayer SrO planes. Surprisingly, we find a disproportionate distribution of La between these layers with the interlayer La being primarily responsible for the IMT. This reveals the distinct site-dependent effects of dopants on the electronic properties of bilayer systems. Electron doping also results in charge reordering. We find unidirectional electronic order concomitant with the structural distortion known to exist in this system. Intriguingly, similar to the single layer iridate, we also find local resonant states forming a checkerboard-like pattern trapped by La. This suggests that multiple charge orders may exist simultaneously in Mott systems, even with only one band crossing the Fermi energy.

npj Quantum Materials (2019)4:43; https://doi.org/10.1038/s41535-019-0183-y

\section{INTRODUCTION}

When holes or electrons are introduced into a Mott insulator, such as the parent compound of high temperature superconducting cuprates, various competing electronic orders emerge and lead to a remarkably rich phase diagram. ${ }^{1}$ Despite years of intense studies, there is still no consensus on the origin of these orders and the relationship between them. This is partly because of the difficulties in obtaining high quality crystals in the lower doping regime close to the Mott transition, as well as the highly insulating nature of Mott systems, which makes them prohibitive for local probes like scanning tunneling microscopy (STM). Recently, an intriguing parallel has arisen between the $S=1 / 2$ Mott phases of the cuprate and the spin-orbit coupling driven $J_{\text {eff }}=1 / 2$ Mott states of their heavier, iridium oxide cousins, ${ }^{2-14}$ which opens up a new pathway to unraveling some of the mysteries of the lightly doped Mott insulators.

The Ruddlesden-Popper series of strontium iridates is a homologous series of compounds containing planes of corner sharing $\mathrm{IrO}_{6}$ octahedra separated by rock-salt $\mathrm{SrO}$ spacing layers. The single layer compound, $\mathrm{Sr}_{2} \mathrm{IrO}_{4}$ (Ir214), shares similarities with its copper oxide cousin, $\mathrm{La}_{2} \mathrm{CuO}_{4}$, not only in the Mott insulating antiferromagnetic (AFM) parent state, ${ }^{2-6}$ but also in the novel electronic states emerging upon carrier doping, such as Fermi arcs, $^{7}$ d-wave gaps, $^{8-10}$ stripy orders, ${ }^{11,12}$ and a pseudogap phase. ${ }^{7,10,11,13}$ The bilayer $\mathrm{Sr}_{3} \mathrm{Ir}_{2} \mathrm{O}_{7}$ (Ir327) retains the $J_{\text {eff }}=1 / 2$
Mott state while lying close to an insulator-metal transition. ${ }^{14}$ Distinct from Ir214, Ir327 is composed of stacked bilayer-IrO ${ }_{6}$ octahedra, which share a SrO charge reservoir layer in between, sandwiched by SrO planes on either side (Fig. 1a). The multiplicity of charge reservoir layers offers a new variation in the dopant position. Specifically, an A-site dopant on the top/bottom layer (Sr1) is in a very different environment from an A-site dopant in the middle layer (Sr2) (Fig. 1a). The potentially disparate effects of doping into these layers have not yet been studied at the microscopic scale.

When $\mathrm{Sr}^{2+}$ is substituted with $\mathrm{La}^{3+}$, the system undergoes a first order transition to a correlated metallic state, ${ }^{15-17}$ where a structural distortion, namely an alternating zigzag tilting of the oxygen octahedra, has been observed. ${ }^{18}$ In addition, based on the low frequency mode in the optical reflectivity measurements, the existence of an electronic density wave (DW) has been postulated. ${ }^{19}$ In this work, we use STM and spectroscopy to study the insulator-to-metal transition (IMT) and possible charge order in the electron-doped metallic state of $\left(\mathrm{Sr}_{1}-\mathrm{x} \mathrm{La}_{\mathrm{x}}\right)_{3} \mathrm{Ir}_{2} \mathrm{O}_{7}$ with $x=$ $0.032,0.039$, and 0.048 . We find that the dopants at two inequivalent $\mathrm{Sr}$ sites have surprisingly distinct effects on the electronic properties: it is predominantly the interlayer La that induces the IMT, which proceeds through a transfer of local spectral weight into the Mott gap. In addition, we find evidence for both a local short-range as well as a long-range periodic rearrangement of charge of different wavelengths. The latter has

\footnotetext{
${ }^{1}$ Department of Physics and Frederick Seitz Materials Research Laboratory, University of Illinois Urbana-Champaign, Urbana, IL 61801, USA; ${ }^{2}$ Department of Physics, Boston College, Chestnut Hill, MA 02467, USA; ${ }^{3}$ Center for Nanoscale Science and Technology, National Institute of Standards and Technology, Gaithersburg, MD 208993, USA and ${ }^{4}$ Materials Department, University of California, Santa Barbara, CA 93106, USA

Correspondence: Vidya Madhavan (vm1@illinois.edu)

These authors contributed equally: Zhenyu Wang, Daniel Walkup
}

Received: 9 January 2019 Accepted: 18 July 2019

Published online: 09 August 2019 


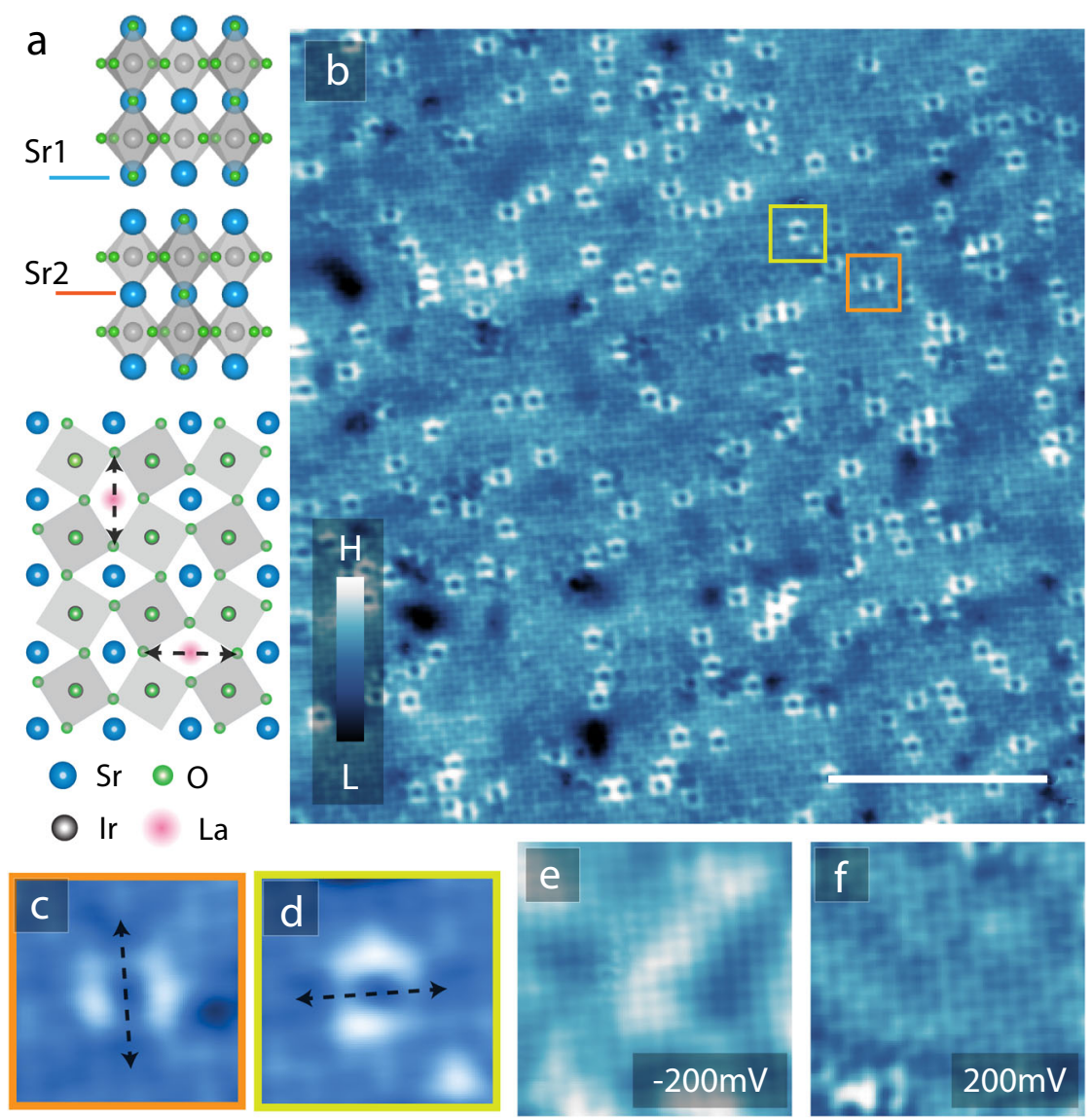

Fig. 1 Surface La dopants. a Crystal structure and schematic of the $\mathrm{IrO}_{6}$ octahedral rotation in $\mathrm{Sr}_{3} \mathrm{Ir}_{2} \mathrm{O}_{7}$. b Atomic-resolution topography on $x$ $=0.032 \mathrm{La}$-doped sample. $V_{S}=200 \mathrm{mV}, l_{t}=100 \mathrm{pA}$. Scale bar: $10 \mathrm{~nm}$. c, $\mathbf{d}$ Zoom-in on two types of La defects in $\mathbf{b}$ showing the reduction of local C4 rotation symmetry to C2. e, $\mathbf{f}$ Topographies taken with different bias voltages in a $6.5 \mathrm{~nm}$ by $6.5 \mathrm{~nm}$ area

the same symmetry and ordering vector as the structural distortion found upon electron doping, suggesting that the frozen phonons go hand in hand with the charge ordering observed in our STM studies.

\section{RESULTS AND DISCUSSION}

Site-dependent effects of La dopants

The crystals cleave easily between two adjacent SrO layers, exposing $\mathrm{SrO}$ terminated surfaces. Figure $1 \mathrm{~b}$ depicts a topographic image taken on the $x=0.032$ sample, and it represents several typical features in these compounds. A square lattice with lattice constant $a=3.9 \AA$, as well as atomic-scale features in the form of bright squares of size $2 \mathrm{a}$, which were absent in previously studied parent samples ${ }^{14}$ are observed. We identify these as La dopants. Consistent with La being substitutional dopants, the centers of the dopants lie on the observed Sr lattice. The La atoms substituting for the top layer Sr1 sites are henceforth called La1. Zooming into the La dopants the density of states around each defect is dimorphic, appearing like two parallel lines instead of a square (Fig. 1C, d). As shown in Fig. 1a, this broken C4 symmetry arises from the local symmetry of the underlying lattice. The staggered rotation of the $\mathrm{IrO}_{6}$ octahedra about c-axis make two adjacent $\mathrm{Sr}$ sites in the same $\mathrm{SrO}$ plane inequivalent: for one site the closest $\mathrm{O}$ atoms in the $\mathrm{IrO}_{2}$ plane are along $a$-axis, while for the other they are along $b$-axis. Thereby, the immediate environment of a $\mathrm{Sr}$ site preserves reflection symmetry, but locally reduces C4 rotation symmetry to $\mathrm{C} 2$. We find that the two different orientations of the density of states signatures of La1, are perfectly correlated with the expected octahedral rotation for a large field of view (see
Supplemental information (SI) note 1) thereby establishing the long-range ordered nature of the octahedral rotations in the doped samples. $^{14}$

A visual examination of the topography reveals that the density of La1 does not correspond to the nominal doping. In fact, a count of the number of La1 gives an areal density corresponding to a nominal doping of only $\sim 1.5 \%$ (assuming a uniform distribution across the three SrO layers), which is far smaller than the actual doping level of $3.2 \%$ determined via energy dispersive spectroscopy (EDS) measurements. To find the missing La atoms, we obtain atomically resolved $\mathrm{dl} / \mathrm{dV}(r, \mathrm{eV})$ maps at different energies, as shown in Fig. 2a, b. In previous studies of bilayer cuprates, dl/dV $(r$, eV) maps were successfully used to detect buried dopants oxygen dopants. ${ }^{20,21}$ Similarly, our local density of states (LDOS) maps reveal a forest of bright atomic-scale features appearing near $E_{F}$ (Fig. 2b, c). These bright features cannot be accounted for by La1 (blue dots in Fig. 2b) and therefore represent distinct atomic-scale impurities.

To clarify the chemical nature of these atomic-scale features (bright dots), we check their positions with respect to the $\mathrm{Sr}$ lattice. We first apply the Lawler-Fujita drift-correction algorithm ${ }^{22}$ to the atom-resolved topographies and $\mathrm{dl} / \mathrm{dV}$ maps. This process removes the warping caused by piezo relaxation and thermal drift. A zoomed-in image of the area marked by the white square in Fig. $2 b$, is shown in Fig. 2c. Visual inspection shows that most of the bright dots line up with the Sr lattice. To confirm this for a statistically significant number of impurities, we obtain the coordinates of the centers of all bright dots in a $20 \mathrm{~nm} \times 20 \mathrm{~nm}$ area. These are plotted with respect to the lattice in Fig. $2 \mathrm{~d}$. We find that within the error represented by the resolution, the vast 
a

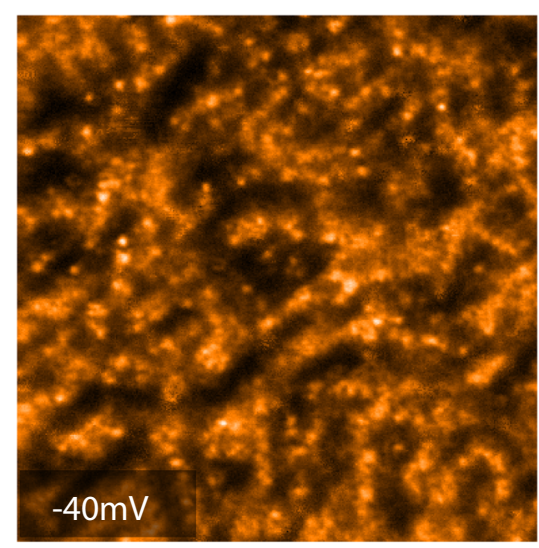

b

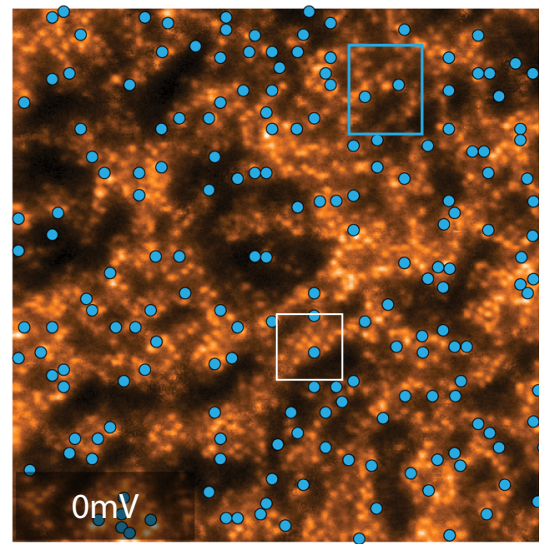

e
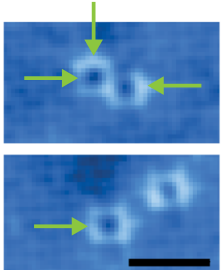

C
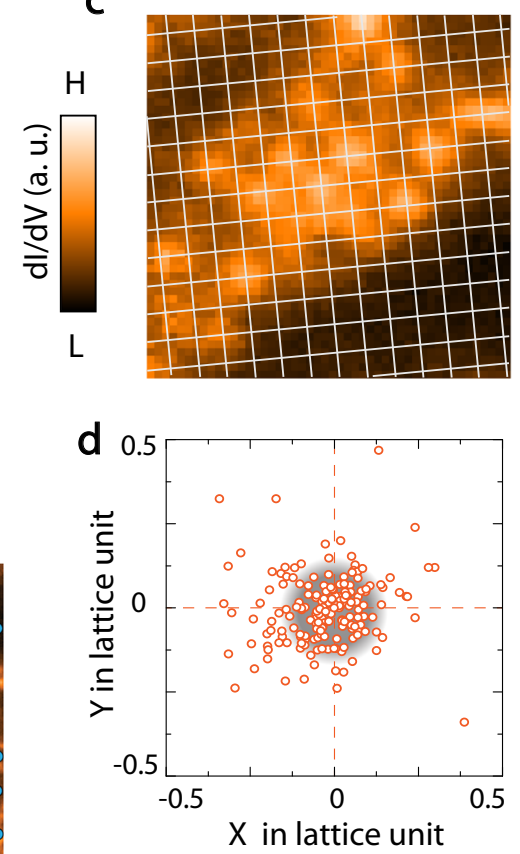

f

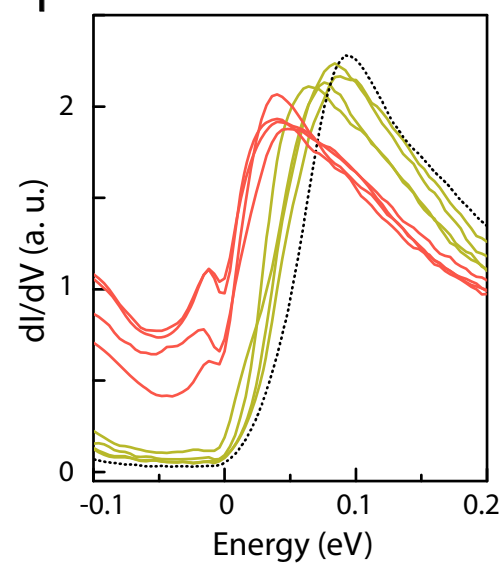

Fig. 2 Middle-layer La dopants. $\mathbf{a}, \mathbf{b} \mathrm{dl} / \mathrm{dV}$ maps taken in the same region of Fig. $1 \mathrm{~b}$, at $\mathrm{E}=-40$ and 0 meV. Clear atomic-scale features appear at the Fermi level $(E=0 \mathrm{meV})$. The blue dots denote the surface La1 dopants. $\mathbf{c}$. Zoom-in of the area marked by white square in $\mathbf{b}$. The white lines denote the Sr lattice obtained from the simultaneously acquired topography. d. A scatter plot showing the positions of the bright features seen in the $\mathrm{dl} / \mathrm{dV}$ map in lattice coordinates. The Sr position is set to $(0,0)$. e. Images of La1 and La2. Arrows mark the positions where the tunneling spectra in $\mathbf{f}$ were obtained. Scale bar: $2 \mathrm{~nm}$. f. Tunneling spectra on La1 (green) and La2 (pink) with set point $\mathrm{V}_{\mathrm{S}}=300 \mathrm{mV}, \mathrm{I}_{\mathrm{t}}=$ $1.2 \mathrm{nA}$. The dark dashed curve denotes spectrum taken at a defect free region

majority of the dots are centered at the Sr sites. The position of the bright dots at $\mathrm{Sr}$ sites, combined with the lack of such high intensity spots in the parent compound suggests that these arise from La dopants occupying Sr sites one-layer underneath (the $\mathrm{Sr} 2$ site). These La dopants at the Sr2 sites are henceforth called La2.

This hypothesis that the bright dots correspond to La dopants in $\mathrm{Sr} 2$ sites can be confirmed by counting their number and comparing this to the expected doping concentration from EDS. Assuming all the observed bright features in the $\mathrm{dl} / \mathrm{dV}$ maps are La2, we get the total doping concentration to be $x=2 / 3 \rho_{L a 1}+1 / 3 \rho_{L a 2}$. For the $3.2 \%$ sample, the areal densities for La1 and La2 substitutions are found to be 1.5 and $7.3 \%$, respectively, so one obtains $x=3.4 \%$, which is within error of the EDS results of $3.2 \%$. A similar analysis for the $3.9 \%$ compound (SI note 2 ) gives us a doping level of $4.1 \%$ by counting. While there is a possibility that the La2 atoms occupy an interstitial site that coincidently lies right under the Sr sites, we believe that this is unlikely based on a comparison with the La-doped single layer Ir214 where a count of the top La1 seen in the topography corresponds well to the nominal doping (SI note 2). Since the only difference between the single and bilayer compounds is that the middle-layer $\mathrm{Sr} 2$ site does not exist in the single layer, this provides further supporting evidence for La2 being substitutional dopants at the Sr2 sites. From the data, the density of La2 (7.3\%) is more than double the outer two layers put-together (3\%). This is an unexpected finding and indicates that La dopants preferentially occupy the $\mathrm{Sr} 2$ sites in the middle layer of the bilayer structure.

Having identified the two doping sites leads to a natural question: Do La1 and La2 have the same effect on the density of states? Spectra on a few different La1 and La2 impurities (Fig. 2e) are shown in Fig. 2f. Comparing the spectra far from impurities (black dashed curve) to the spectra on La1 impurities (dark green), we see that La1 has little effect on the density of states except to slightly move the leading-edge position of the conduction band. 

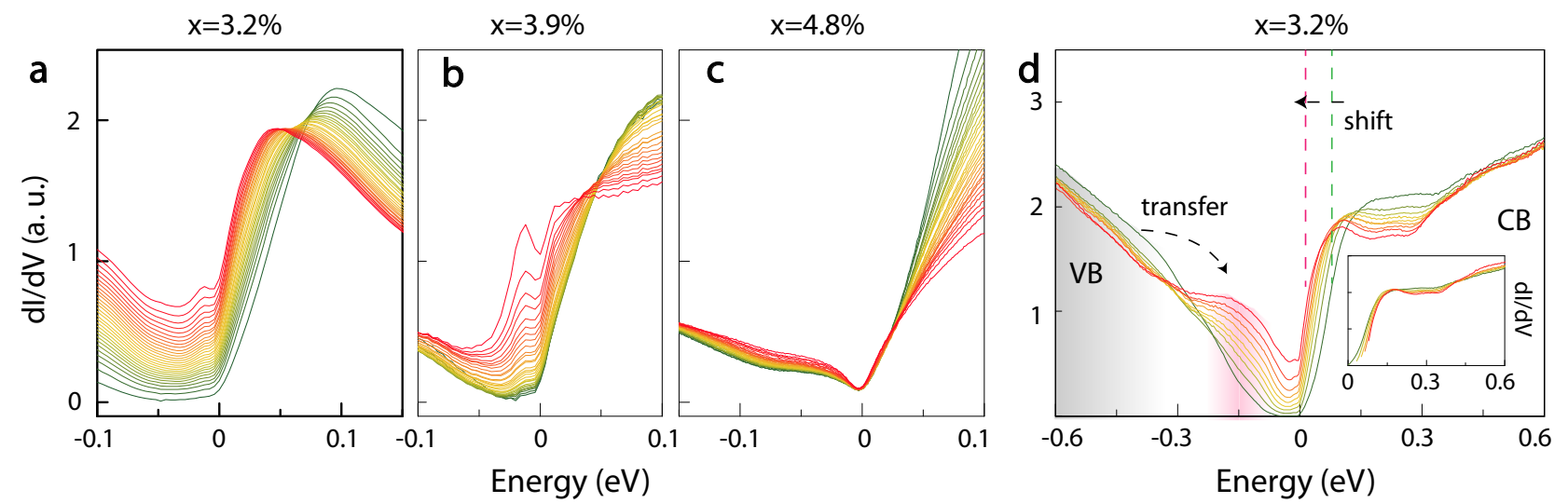

Fig. 3 Evolution of tunneling spectra across IMT. a-c Binned spectra for $x=0.032,0.039$, and 0.048 samples. Differential conductance at -40 , -40 , and $100 \mathrm{meV}$ were chosen to classify the spectra for $\mathbf{a}-\mathbf{c}$, respectively. Then the spectra are split into 30 groups with equal population. Green/red curves denote more insulating/metallic behavior in a and $\mathbf{b}$. $\mathbf{d}$ binned spectra in a larger energy range on the $x=0.032$ samples, based on the differential conductance at $0 \mathrm{mV}$. Inset: normalized spectra with shifted energy to compare the overall line shape above Fermi level. Set point: $160 \mathrm{mV}, 800 \mathrm{pA}$ for a; $100 \mathrm{mV}, 180 \mathrm{pA}$ for $\mathbf{b}$; $-300 \mathrm{mV}, 700 \mathrm{pA}$ for $\mathbf{c}$, and $600 \mathrm{mV}, 4 \mathrm{nA}$ for d

The effect of La2 (pink) however, is much more dramatic, creating a large density of states within the gap (also see line-cut profiles across La1 and La2 in SI note 3).

To better visualize overall trends in the $\mathrm{dl} / \mathrm{dV}$ spectra, we present 'binned-averaged spectra' in the Fig. 3. The spectra obtained in a $\mathrm{dl} / \mathrm{dV}$ map are first binned against the density of states value at $-40 \mathrm{meV}$, which captures the inhomogeneity in the sample (see dl/dV $(r,-40 \mathrm{meV})$ map in Fig. 2a). We then average all spectra in each bin resulting in the binned-averaged spectra shown in Fig. 3 (for more details see SI note 4). In the $x=$ 0.032 sample (Fig. 3a), the green curves corresponding to the dark regions in $\mathrm{dl} / \mathrm{dV}(r, \mathrm{eV})$ exhibit fully insulating line shapes reminiscent of the parent compound with a gap up to $100 \mathrm{meV}$. In stark contrast, the red curves show significant nonzero density of states at the Fermi energy. By comparing the shape of the red curves with spectra obtained on individual La1 and La2 impurities shown in Fig. $2 f$, we can see that the red curves and La2 spectra have almost identical line shapes, which suggest that the subsurface La2 cause the increase in the density of states at the Fermi energy that leads to the IMT. This is confirmed by a correlation analysis between the locations of La2 impurities and the density of states map, which shows a correlation coefficient of 0.75 . The analysis also shows negligible correlation between La1 and the density of states distribution (SI note 5). Thus, we deduce that the subsurface La2 are primarily responsible for the doping induced insulator-metal transition.

The binned-averaged spectra allow us to make a few general observations on the effect of electron doping on the electronic structure. The parent compound hosts an insulating gap $\sim 130$ meV with the Fermi energy located near the top of valence band. ${ }^{14}$ Consistent with La being an electron donor, upon doping, the Fermi level overall moves up about $100 \mathrm{mV}$ to the bottom of the conductance band (SI note 6). In the 0.032 and 0.039 samples there are still areas with insulating gaps although the largest gap magnitude observed has decreased significantly to $30 \mathrm{meV}$ in the 0.039 compound. In the metallic samples, all spectra are gapless and show a similar overall spectral shape. We now concentrate on the 0.032 sample, and start the discussion with the position dependent changes of the conduction band edge near $\mathrm{E}_{\mathrm{F}}$. As shown in Fig. 3d, in areas with larger local densities of La2, the midpoint of conduction band leading edge moves towards the Fermi energy. However, the spectral shape of the conduction band does not change significantly (see inset to Fig. 3d where we have simply shifted the spectra in energy such that they lie on top of each other), suggesting the conduction band motion mimics a local potential variation or a local rigid band shift. This is consistent with photoemission data, which show that with increasing $\mathrm{La}$, the Fermi energy moves into the conduction band at the $M$ point. ${ }^{16,23}$ In a simple picture, the spatially varying leading-edge positions seen in STM data may be construed as an inhomogeneous distribution of charge causing potential variations about the mean doping level (SI note 7).

However, this is not the only effect of La2. As the local density of La increases, there is increasing spectral weight accumulation around $-150 \mathrm{meV}$ (Fig. 3d), close to the hopping energy $t=$ $0.2 \mathrm{eV}^{24}$ Simultaneously, the density of states at deeper energies (below $-300 \mathrm{meV}$ ) is suppressed. Thus, the subsurface La is associated with a spectral weight transfer from the valance band towards the Fermi energy. This kind of spectral weight transfer is different from electron doping caused by missing anions on the surface, ${ }^{14,25}$ where only the upper Hubbard band is involved, but is consistent with the theoretical models proposed for electrondoped cuprates. $^{26}$ It has been suggested that in the AFM insulating background, upon electron doping, the creation of particle-hole spin excitations in the upper band strongly admixes the quasi-hole states in the upper and lower bands, giving rise to the emergence of spectral weight at an energy of approximately $-t$ in the charge gap. In $\left(\mathrm{Sr}_{1-x} \mathrm{La}_{\mathrm{x}}\right)_{3} \mathrm{Ir}_{2} \mathrm{O}_{7}$, despite the destruction of static AFM, magnetic excitations can persist across the IMT. ${ }^{15,17}$ Whether or not this treatment captures the nature of $\left(\mathrm{Sr}_{1-x} \mathrm{La}_{\mathrm{x}}\right)_{3} \mathrm{Ir}_{2} \mathrm{O}_{7}$, the spectral weight transfer from the valence bands is a distinct aspect of electron doping in this system.

We now address possible reasons for distinct effects of La1 and La2 on the density of states. In Ir214, it was found that for low dopings below the IMT, the La atoms have a minimal effect on the LDOS due to Mott localization. ${ }^{11}$ This picture breaks down for dopings above $4 \%$. (Note that Ir 214 is a single layer compound and therefore has only one site for La dopants). One could extrapolate from that study and speculate that the different behavior of La1 and La2 is due to their different densities. In this scenario, the top layer with only $1.5 \%$ La behaves like the Ir214 sample below the IMT where the electrons are localized due to strong correlations. However, this scenario is not completely applicable to the bilayer compound. Here, electrons from La2 are expected to reside in the $\mathrm{IrO}_{2}$ planes, where they would contribute to screening the top SrO plane, thereby reducing this localization effect. The source of the difference between La1 and La2 is therefore not completely obvious and further experimental and theoretical work is necessary to pinpoint the origin of this dichotomy. 

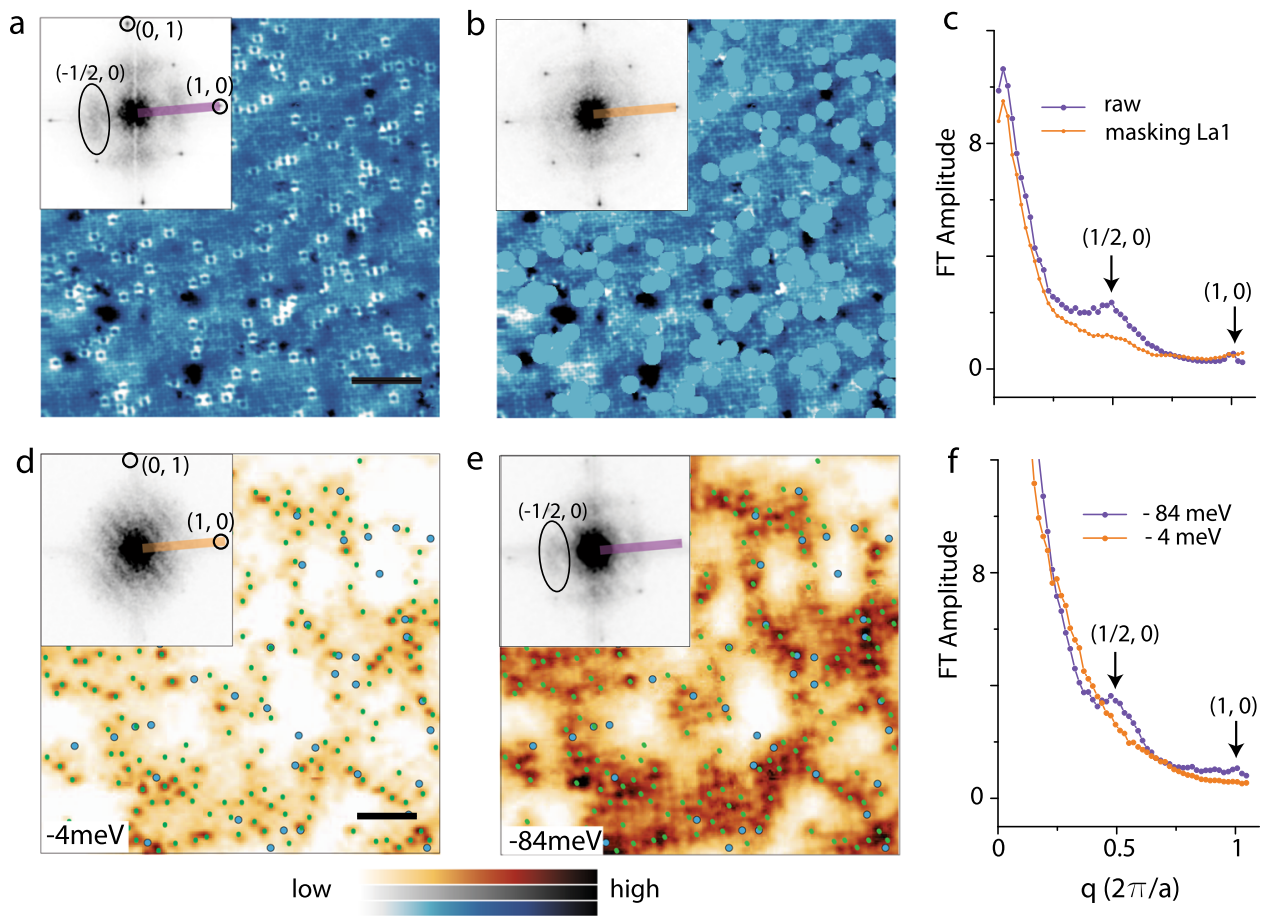

Fig. 4 Features in the FT at $(1 / 2,0)$. a Atomic-resolution topography of the $x=0.032$ sample (same as Fig. $1 \mathrm{~b}$ ). The inset shows its FT. $(0,1)$ and $(1,0)$ are the $\mathrm{Sr}$ reciprocal lattice vectors. Scale bar: $7 \mathrm{~nm}$. b Masked topography to remove the La1 features in a and its FT. $\mathbf{c}$ Linecuts along $(1$, 0) direction of the FT before and after masking La1. d, e dl/dV map at -4 and $-84 \mathrm{meV}$, respectively. The blue and green dots denote the position of La1 and La2, respectively. dl/dV map at $-84 \mathrm{meV}$ shows glassy checkerboard-like modulation, clearly centered around the locations of La2. Scale bar: $3 \mathrm{~nm}$. $\mathbf{f}$ Linecuts along $(1,0)$ direction of the FTs shown in the insets of $\mathbf{d}$ and $\mathbf{e}$

Revealing potential electronic order

We now turn to the question of possible charge ordering in this system. A DW order was recently discovered in this system, ${ }^{19}$ which emerges upon electron doping and forms concomitantly with the tilting of the oxygen octahedra. ${ }^{18}$ Recent studies on the electron-doped Ir $214^{11}$ reveal a disordered density of states modulation with 2 a period, which was interpreted in terms of a local stripe order resembling the 4a modulation in underdoped cuprates. ${ }^{27,28}$ The question then arises whether a similar order is seen in $\left(\mathrm{Sr}_{1-x} \mathrm{La}_{\mathrm{x}}\right)_{3} \mathrm{Ir}_{2} \mathrm{O}_{7}$. Examining the Fourier transforms (FTs) of the topography, as well as the $\mathrm{dl} / \mathrm{dV}$ maps, we find that in addition to the Bragg peaks, there are peaks at the $( \pm 1 / 2, \pm 1 / 2)$ positions, as well as a diffuse intensity around the $( \pm 1 / 2,0)$ and $(0, \pm 1 / 2)$ positions (Fig. 4). The peaks at $( \pm 1 / 2, \pm 1 / 2)$ also exist in the parent compound due to the alternating octahedral rotations and are not unique to the La-doped samples. The diffuse $( \pm 1 / 2,0)$ signal however indicates a real space pattern with 2 a periodicity along the Ir-O-Ir direction.

A probable source of the $( \pm 1 / 2,0)$ feature in the FT of the topography is the La1 induced impurity resonance (Fig. 4a), which is observed as a square shaped density of states of period $2 a$ around the La1 atoms. This can be confirmed by masking these features (Fig. 4b), whereupon the $( \pm 1 / 2,0)$ feature in the FT of the topography disappears (Fig. 4c). The occurrence of the $( \pm 1 / 2,0)$ intensity in the FT of the $\mathrm{dl} / \mathrm{dV}$ maps (Fig. $4 \mathrm{f}$ ) is however subtler. In fact, we find that the $\mathrm{dl} / \mathrm{dV}$ maps also show stripe like periodic patterns, which are now correlated with La2. We can see this by comparing the positions of the La2 shown as green dots (Fig. 4e) with the stripy pattern in real space. This indicates that the La2 atoms trap the same local 2a period resonance states as the La1 atoms. Unlike previous work where no connection was established between the La induced resonance states ${ }^{11}$ and the glassy charge order, our data indicate that the La1 and La2 induced localized resonance states are responsible for much of the period $2 a$ signatures in real and Fourier space. An energy dependence of the correlation between La dopants and LDOS can be found in SI note 8. The connection between such local impurity resonance states and an incipient charge DW instability remains an open interesting question. However, this signal is suppressed in the metallic sample and may therefore be unrelated to the DW instability ${ }^{19}$ observed in this compound, which persists far into the metallic phase.

There is however another potential charge order in this system. With La doping, we find that the topographies at different bias voltages become strongly energy dependent. At high negative bias voltages, the expected square lattice for $\mathrm{Sr}$ is observed (Fig. 1e). This suggests the Sr lattice remains undistorted despite the tilting of the oxygen octahedra. However, at other energies, a zigzag pattern is observed (Figs. If and S11g). Because topographic images contain information on both the surface corrugation as well as LDOS, the energy dependent topography we see here likely reflects charge rearrangement due an underlying electronic order. To visualize this electronic order, we utilize a supercell averaging algorithm, which has been successfully applied to cuprates. ${ }^{29}$ The supercell algorithm is an averaging technique that utilizes the spatially dependent topography to construct an averaged unit cell that reflects any long-range ordered structure. From a series of bias dependent topographies on multiple samples, we plot the locations where maximal integrated DOS appears within unit cell as shown in Fig. $5 \mathrm{~d}$ and also in the SI note 9.

The average unit cell topographies at high negative energies reveal Sr atoms at the expected positions of the lattice (crosses in Fig. 5d). However, the data in a band of low energies (below and above $E_{F}$ ) show a consistent shift of the LDOS with respect to $\mathrm{Sr}$ sites in alternate directions along the diagonal direction (Ir-Ir direction). This pattern, visually seen as a zigzag pattern (Fig. S11), represents the property of an underlying electronic order, which breaks the local two-dimensional inversion symmetry in the $\mathrm{IrO}_{2}$ plane. Interestingly, the structural distortion, stemming from the 
a

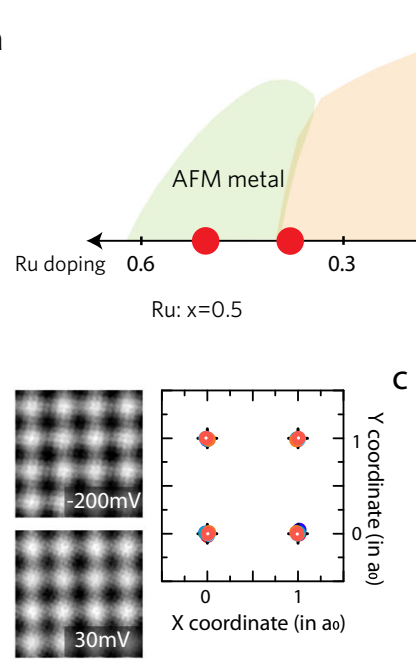

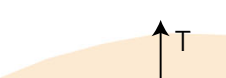

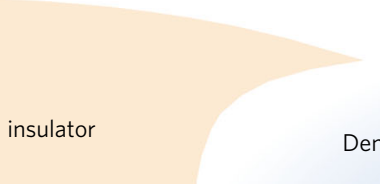

0.02

La: $x=0.032$

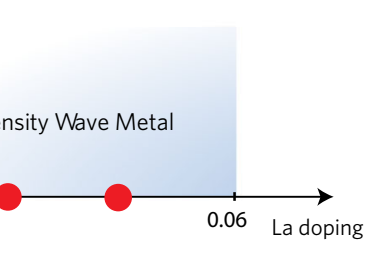

e

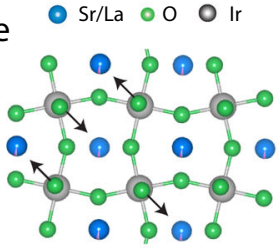

d
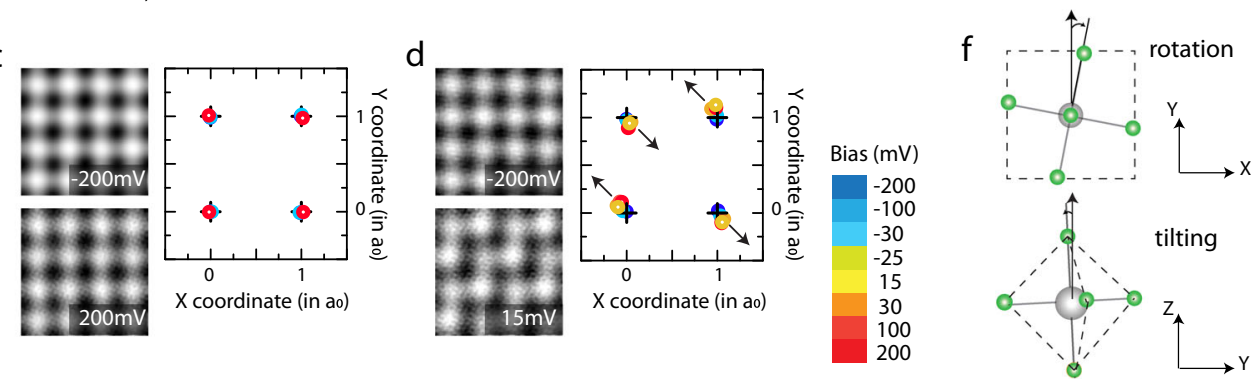

Fig. 5 Visualizing the density wave. a Schematic phase diagram for La- and Ru-doped Ir327. Red dots represent the samples studied here. b-d Energy dependence of the averaged supercell $(4 \times 4$ blocks) for the parent compound, Ru-doped and La-doped samples. The center of visible "atom" is fit by 2D Gaussian function and plotted in unit cell coordinate e Schematic of the IrO $_{6}$ octahedra tilting in La-doped samples $(x=$ 0.035). $\mathbf{f}$ cartoon images showing the in-plane rotation and off-plane tilting of the $\mathrm{IrO}_{6}$ octahedra in La-doped $\mathrm{Ir} 327$

tilting of $\mathrm{IrO}_{6}$ octahedra ${ }^{18}$ has the same symmetry as the electronic order we find here (Fig. 5e). To confirm that this order is tied to the tilting, we perform a comparative supercell analysis on the Ru-doped (B-site dopant) and pristine compounds where neither the DW nor the tilting has been observed. Correspondingly, the supercells in these compounds show no energy dependence (Fig. 5b, c and supplemental Fig. S12), and all the visible atoms are located at perfect square lattice sites as expected from the Sr lattice. In combination with the optical spectroscopy results, our data across the pristine, $\mathrm{Ru}$-, and La-doped compounds indicate that this local inversion symmetry breaking charge order is intertwined with both the structural distortion and the DW.

In summary, our measurements reveal the microscopic mechanism for the IMT and formation of DW states within the $J_{\text {eff }}=1 / 2$ state in $\left(\mathrm{Sr}_{1}{ }_{-x} \mathrm{La}_{\mathrm{x}}\right)_{3} \mathrm{Ir}_{2} \mathrm{O}_{7}$. La dopants preferentially occupy the $\mathrm{Sr}$ sites in the middle plane of iridium oxide bilayers, inducing a spectral weight transfer from the lower Hubbard band to a new low-energy quasiparticle band. This leads to local electron density fluctuations that promote nanoscale phase separation prior to the formation of a global metallic state. The extended Coulomb interactions between electrons in the spatially large Ir $5 \mathrm{~d}$ orbitals could play an important role. ${ }^{6}$ Within the lowenergy band, multiple electronic orders emerge. A unidirectional order, which breaks local inversion symmetry, is absent in both the pristine, and B-site substituted samples, demonstrating that the charge instability is endemic to electron doping in this system. This primary charge order parameter intertwines with the previously reported structural distortion known to onset below $200 \mathrm{~K}$. We additionally observe local resonant states forming a checkerboard-like pattern trapped by $\mathrm{La}$, which were also previously observed in $\mathrm{Sr}_{2} \mathrm{IrO}_{4}{ }^{11}$ Our combined data show a microscopic picture of how the weak Mott ground state in a bilayer iridate collapses with electron doping and indicates a potential coexistence of two types of order within one $J_{\text {eff }}=1 /$ 2 band.

\section{METHODS}

\section{STM measurement}

Single crystals of $\left(\mathrm{Sr}_{1-\mathrm{x}} \mathrm{La}_{\mathrm{x}}\right)_{3} \mathrm{Ir}_{2} \mathrm{O}_{7}$ were grown via flux techniques reported earlier, ${ }^{30}$ and the actual La concentrations were confirmed by EDS. The IMT happens at $x=0.04$ and a detailed phase diagram can be found elsewhere. ${ }^{15}$ All samples are cleaved at $\sim 77 \mathrm{~K}$ in ultra-high vacuum and immediately inserted into the STM head where they are held at $\sim 4.3 \mathrm{~K}$ during the process of data acquisition. Differential conductance maps $\mathrm{dl} /$ $\mathrm{dV}(r, \mathrm{eV})$ and spectra $\mathrm{dl} / \mathrm{dV}(\mathrm{eV})$, which are proportional to the LDOS at position $r$ and energy eV, were obtained using standard lock-in technique.

\section{Average supercell algorithm}

First, we use the drift-correction method ${ }^{22}$ to remove the slow thermal drift and warping from piezo relaxation in the topographic images. The atoms are placed at the true lattice positions after this treatment. Then we create a blank $2 \times 2$ supercell with better spatial resolution than the raw data. In this work, it is $25 \times 25$ pixels per unit cell. For each pixel in the driftcorrected topographies, we calculate its position in lattice coordinate, and then place it in the appropriate bin of the supercell. A detailed description of this method can be found. ${ }^{29}$ After $2 \times 2$ supercells obtained, we tile them two times to create a larger supercell for better visualization, as shown in Fig. 5.

\section{DATA AVAILABILITY}

The data sets that support the findings in this study are available from the corresponding author upon reasonable request.

\section{ACKNOWLEDGEMENTS}

We thank Ilija Zeljkovic, Hsin Lin, Eduardo Fradkin, and Milan Allan for helpful discussions. V.M. gratefully acknowledges NSF Award No. DMR-1610143 for the STM studies. S.D.W. and T.H. acknowledge funding support from NSF Award No. DMR1505549. Z.W. is supported by the U.S. Department of Energy, Basic Energy Sciences Grant No. DE-FG02-99ER45747.

\section{AUTHOR CONTRIBUTIONS}

Z.W. and D.W. contributed equally to this work. V.M., S.D.W. and Z.W. conceived the experiments. D.W. and Z.W. obtained the STM data. T.H. and S.D.W. were responsible for sample growth and characterization. V.M., Z.W., Y.M., and D.W. performed data analysis. All authors participated in preparing the manuscript.

\section{ADDITIONAL INFORMATION}

Supplementary information accompanies the paper on the npj Quantum Materials website (https://doi.org/10.1038/s41535-019-0183-y).

Competing interests: The authors declare no competing interests. 
Publisher's note: Springer Nature remains neutral with regard to jurisdictional claims in published maps and institutional affiliations.

\section{REFERENCES}

1. Keimer, B., Kivelson, S. A., Norman, N. R., Uchida, S. \& Zaanen, J. From quantum matter to high-temperature superconductor in copper oxides. Nature $\mathbf{5 1 8}$, 179-186 (2015)

2. Kim, B. J. et al. Novel $J_{\text {eff }}=1 / 2$ Mott state induced by relativistic spin-orbit coupling in $\mathrm{Sr}_{2} \mathrm{IrO}_{4}$. Phys. Rev. Lett. 101, 076402 (2008).

3. Kim, J. et al. Magnetic excitation spectra of $\mathrm{Sr}_{2} \mathrm{IrO}_{4}$ probed by resonant inelastic Xray scattering: Establishing links to cuprate superconductors. Phys. Rev. Lett. 108, 177003 (2012).

4. Rau, J. G., Lee, E. K. \& Kee, H. Spin-orbit physics giving rise to navel phase in correlated systems: iridates and related materials. Annu. Rev. Condens. Matter Phys. 7, 195-221 (2016).

5. Wang, F. \& Senthil, T. Twisted Hubbard model for $\mathrm{Sr}_{2} \mid \mathrm{IO}_{4}$ : magnetism and possible high temperature superconductivity. Phys. Rev. Lett. 106, 136402 (2011).

6. Zhou, S., Jiang, K., Chen, H. \& Wang, Z. Correlation effects and hidden spin-orbit entangled electronic order in parent and electron-doped iridates $\mathrm{Sr}_{2} \mathrm{IrO}_{4}$. Phys. Rev. X 7, 041018 (2017).

7. Kim, Y. K. et al. Fermi arcs in a doped pseudospin-1/2 Heisenberg antiferromagnet. Science 345, 187-190 (2014).

8. Kim, Y. K., Sung, N. H., Denlinger, J. D. \& Kim, B. J. Observation of a d-wave gap in electron-doped $\mathrm{Sr}_{2} \mathrm{IrO}_{4}$. Nat. Phys. 12, 37-41 (2016).

9. Yan, Y. J. et al. Electron-doped $\mathrm{Sr}_{2} \mid \mathrm{rO}_{4}$ : an analogue of hole-doped cuprate superconductors demonstrated by scanning tunneling microscopy. Phys. Rev. X 5, 041018 (2015).

10. de la Torre, A. et al. Collapse of the Mott gap and emergence of a nodal liquid in lightly doped $\mathrm{Sr}_{2} \mathrm{IrO}_{4}$. Phys. Rev. Lett. 115, 176402 (2015).

11. Battisti, I. et al. Universality of pseudogap and emergent order in lightly doped Mott insulator. Nat. Phys. 13, 21-25 (2017).

12. Chen, $X$. et al. Unidirectional spin density wave state in metallic $\left(\mathrm{Sr}_{1-\mathrm{x}} \mathrm{La}_{\mathrm{x}}\right)_{2} \mathrm{IrO}_{4}$ Nat. Comm. 9, 103 (2018)

13. Cao, Y. et al. Hallmarks of the Mott-metal crossover in the hole doped pseudospin-1/2 Mott insulator $\mathrm{Sr}_{2} \mathrm{IrO}_{4}$. Nat. Comm. 7, 11367 (2016).

14. Okada, Y. et al. Image the evolution of metallic states in a correlated iridate. Nat. Mater. 12, 707-713 (2013).

15. Hogan, T. et al. First-order melting of a weak spin-orbit Mott insulator into a correlated metal. Phys. Rev. Lett. 114, 257203 (2015).

16. He, J. et al. Spectroscopic evidence for negative electronic compressibility in a quasi-three-dimensional spin-orbit correlation metal. Nat. Mater. 14, 577-582 (2015).

17. Lu, X. et al. Doping evolution of magnetic order and magnetic excitations in $\left(\mathrm{Sr}_{1}\right.$ $\left.\mathrm{La}_{\mathrm{x}}\right)_{3} \mathrm{Ir}_{2} \mathrm{O}_{7}$. Phys. Rev. Lett. 118, 027202 (2017).
18. Hogan, T., Wang, X., Chu, H., Hsieh, D. \& Wilson, S. D. Doping-driven structural distortion in the bilayer iridate $\left(\mathrm{Sr}_{1-x} \mathrm{La}_{x}\right){ }_{3} \mathrm{Ir}_{2} \mathrm{O}_{7}$. Phys. Rev. B 95, 174109 (2017)

19. Chu, H. et al. A charge density wave-like instability in a doped spin-orbit-assisted weak Mott insulator. Nat. Mater. 16, 200-203 (2017).

20. McElroy, K. et al. Atomic-scale sources and mechanism of nanoscale electronic disorder in $\mathrm{Bi}_{2} \mathrm{Sr}_{2} \mathrm{CaCu}_{2} \mathrm{O}_{8+\sigma}$. Science 309, 1048-1052 (2005).

21. Zeljkovic, I. et al. Imaging the impact of single oxygen atoms on superconducting $\mathrm{Bi}_{2+\mathrm{y}} \mathrm{Sr}_{2-\mathrm{y}} \mathrm{CaCu}_{2} \mathrm{O}_{8+\mathrm{x}}$. Science 337, 320-323 (2012).

22. Lawler, M. J. et al. Intra-unit-cell electronic nematicity of high-Tc copper-oxide pseudogap states. Nature 466, 347-351 (2010)

23. de la Torre, A. et al. Coherent quasiparticles with a small Fermi surface in lightly doped $\mathrm{Sr}_{3} \mathrm{Ir}_{2} \mathrm{O}_{7}$. Phys. Rev. Lett. 113, 256402 (2014).

24. Carter, J.-M. \& Kee, H.-Y. Microscopic theory of magnetism in $\mathrm{Sr}_{3} \mathrm{Ir}_{2} \mathrm{O}_{7}$. Phys. Rev. B 87, 014433 (2013).

25. Ye, C. et al. Visualizing the atomic-scale electronic structure of the $\mathrm{Ca}_{2} \mathrm{CuO}_{2} \mathrm{Cl}_{2}$ Mott insulator. Nat. Comm. 4, 1365 (2013).

26. Kusunose, H. \& Rice, T. M. Single-particle spectrum in the electron-doped cuprates. Phys. Rev. Lett. 91, 186407 (2003).

27. Comin, R. et al. Charge order driven by Fermi-Arc instability in $\mathrm{Bi}_{2} \mathrm{Sr}_{2-\mathrm{x}} \mathrm{La}_{\mathrm{x}} \mathrm{CuO}_{6+\sigma}$ Science 343, 390-392 (2014).

28. da Silva Neto, E. H. et al. Ubiquitous interplay between charge ordering and hightemperature superconductivity in cuprates. Science 343, 393-396 (2014).

29. Zeljkovic, I. et al. Scanning tunneling microscopy imaging of symmetry-breaking structural distortion in the bismuth-based cuprate superconductors. Nat. Mater. 11, 585-589 (2012).

30. Dhital, C. et al. Spin ordering and electronic texture in the bilayer iridate $\mathrm{Sr}_{3} \mathrm{Ir}_{2} \mathrm{O}_{7}$. Phys. Rev. B 86, 100401 (2012).

(i) Open Access This article is licensed under a Creative Commons Attribution 4.0 International License, which permits use, sharing, adaptation, distribution and reproduction in any medium or format, as long as you give appropriate credit to the original author(s) and the source, provide a link to the Creative Commons license, and indicate if changes were made. The images or other third party material in this article are included in the article's Creative Commons license, unless indicated otherwise in a credit line to the material. If material is not included in the article's Creative Commons license and your intended use is not permitted by statutory regulation or exceeds the permitted use, you will need to obtain permission directly from the copyright holder. To view a copy of this license, visit http://creativecommons. org/licenses/by/4.0/.

(c) The Author(s) 2019 